\title{
INCOMPATIBILIZAÇÃO DE PROJETOS NA CONSTRUÇÃO CIVIL E SEUS PRINCIPAIS PROBLEMAS.
}

Italo Gutierry Carneiro da Conceição - enggutierrycarneiro@gmail.com*

Instituto Federal do Piauí - IFPI*

Caxias-Maranhão*

Laise Albuquerque Rolim - laise.rolim@gmail.com*

MARKA CONSTRUTORA LTDA e ALBUQUERQUE EMPREENDIMENTOS LTDA*

Teresina-Piauí*

Paulysendra Felipe Silva - sendra717@gmail.com*

Instituto Federal do Piauí - IFPI*

Caxias-Maranhão*

Dhonyzeth Alehf Pereira Barros - dhonyzethpereira@gmail.com*

MPA Construções e Participações*

Bacabal-Maranhão

\section{Resumo}

O artigo teve como objetivo analisar a etapa de desenvolvimento de projetos e a prática de compatibilização dos mesmos, além de verificar as interferências entre os principais tipos de projetos realizados para uma boa execução na construção civil. Ao longo da revisão bibliográfica, são abordados alguns tópicos importantes como conceito e concepção de projeto e sua importância para a construção civil. Logo após, apresentam-se algumas informações sobre compatibilização e coordenação, e por fim, estão presentes alguns tópicos relacionados à plataforma $\mathrm{CAD}$, plataforma BIM e softwares que trabalham com essas tecnologias. Para obter as informações necessárias, optou-se por realizar um levantamento de dados com aplicação de questionário destinado aos profissionais da área de Engenharia Civil e Arquitetura, com questionamentos que enfocam o uso de softwares com metodologia BIM, problemas oriundos da não compatibilização de projetos e aspecto que o BIM mais enfoca. Com os resultados obtidos, foi possível constatar que a quantidade de problemas em edificações devido as interferências entre projetos apresentaram números expressivos. Uma forma de reduzir essa problemática, é através da melhor comunicação entre os profissionais envolvidos na etapa de projeto e execução da obra.

Palavras-chave: Projeto. Construção Civil. Incompatibilização. Coordenação.

\section{Incompatibilization of projects in civil construction and its main problems.}

\begin{abstract}
:
The objective is to analyze the step of projects development and the practice of compatibilization their, besides that, verify a interference between the main types performed for good execution in civil construction. Throughout the bibliographic review presented, some important topics are addressed such as, project concept, as well as its conception process and its importance in the context of the construction, right after, some information about compatibilition and coordination is presented and, finally, they are present some topics related to the CAD platform, BIM platform and software that work with these technologies. In order to obtain the necessary information, it was choosed to carry out a data survey with
\end{abstract}


application of questionnaire destined to the professionals of the Civil Engineering and Architecture area, who work in companies that execute works and elaborate projects of engineering. With this, it will be possible check and relate their combinations and generate data by separating the information into a disposition that guarantees the organization to compile the responses. Finally, the numbers and affirmations of the interviewees will be analyzed so that the conclusions are drawn up.

Keywords: Project. Civil Constructiuon. Incompatibilization. Coordination.

\section{INTRODUÇÃ̃O}

A incompatibilização entre projetos ocasiona muitos problemas na construção civil, tais como, atrasos no cronograma da obra, falhas na execução dos serviços e aumento do custo do empreendimento devido ao retrabalho. Essa pesquisa verificou as medidas necessárias para reduzir tais problemas, através do auxílio de profissionais que tenham experiência em gestão e gerenciamento no setor da construção civil.

A escolha do tema foi justificada visto que a ausência de compatibilização entre projetos tem se tornado um problema frequente que apresenta graves prejuízos para a execução de uma edificação e evidenciado sua detecção de forma regular nas obras. Horostecki (2014) salienta que "compatibilizar projetos requer investimentos que podem representar de $1 \%$ a $1,5 \%$ do custo da obra, mas gera diminuição de despesas que varia de 5\% a 10\% desse mesmo custo".

$\mathrm{O}$ método de pesquisa adotado foi quantitativo, no qual a pesquisa foi dividida em duas etapas: primeiro, foi realizado um levantamento bibliográfico e por último foi realizada uma pesquisa exploratória com profissionais atuantes na construção civil com enfoque na coleta de dados através da aplicação de questionário, para completar o estudo teórico e aproximá-lo do campo concreto de atuação. O local de realização do estudo foi a cidade de Caxias localizada no Estado do Maranhão. Foi constatado que o ponto crítico do surgimento de problemas de execução ocorre devido as interferências entre projetos Estrutural e de Instalações.

\section{OBJETIVOS}

O estudo teve como objetivo geral analisar os principais problemas da incompatibilização de projetos de engenharia e propor meios que os reduzam. Como objetivos específicos: fazer um levantamento bibliográfico sobre o tema de compatibilização de projetos de engenharia; levantar erros de execução entre projetos elaborados em desacordo entre si; avaliar os motivos de incompatibilização entre projetos mais comumente observados; aconselhar e propor medidas alternativas para minimizar a problemática em questão.

\section{RESULTADOS}

Mediante os resultados obtidos através dos questionamentos submetidos aos colaboradores, foi verificado que no panorama atual dos locais onde o estudo foi realizado a superposição de projetos, afim de diminuir as interferências, é realizada em todos os casos com o auxílio de softwares, pois os programas apresentam maior eficiência e melhor aproveitamento de tempo para verificar interferências entre projetos.

Um ponto importante que deve ser abordado é que os problemas oriundos da incompatibilização de projeto têm se tornado tão frequentes que erroneamente os responsáveis técnicos veem os mesmos como uma situação comum nos canteiros de obras.

$\mathrm{Na}$ região onde pesquisa foi realizada, observou-se que os profissionais atuantes no setor construtivo, tem conhecimento sobre a tecnologia CAD e BIM e os benefícios e melhorias que o BIM tem em relação ao $\mathrm{CAD}$, entre elas mencionadas, maior eficiência nos 
planejamentos executados, cumprimento dos cronogramas programados, situações que mais se aproximam da realidade de um canteiro de obra e redução no custo. Percebe-se que devido à realização de tarefas não previstas na parte executiva de um empreendimento, em $60 \%$ dos casos há um aumento no custo final.

Outro ponto importante que foi abordado é que de acordo com os entrevistados, todas as empresas sofreram alguma penalidade em consequência de falhas oriundas por incompatibilização de projetos e mesmo diante disso essa otimização não é desempenhada adequadamente, ou seja, o software utilizado para tal tarefa é baseado na plataforma CAD que possui muitas limitações se comparado ao BIM. No panorama do estudo o Autodesk Autocad é o software que desempenha essa tarefa de compatibilização, mesmo os profissionais tendo conhecimento sobre os softwares quem utilizam a tecnologia BIM, como mostra a figura abaixo:

Figura 1 - Software utilizado para compatibilização de projetos

AUTODESK AUTOCAD

AUTODESK NAVISWORKS

AUTODESK REVIT

TEKLA STRUCTURES

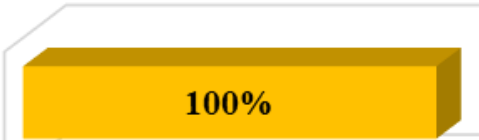

$0 \%$

$0 \%$

$0 \%$

Fonte - Autores (2018)

O cenário obtido mediante os dados acerca da análise de execução de obras, os problemas oriundos de interferências entre projetos de engenharia são encontrados frequentemente. Nota-se que a constante aparição de problemas devida a incompatibilização é comum nos canteiros de obras, como mostra a figura abaixo:

Figura 2 - Aparição de problemas oriundos de incompatibilização de projetos.

RARAMENTE

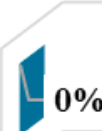

FREQUENTEMENTE

$100 \%$

NUNCA

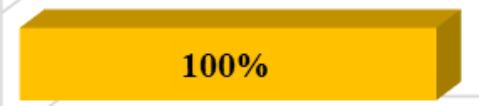

$0 \%$

$0 \%$

Fonte - Autores (2018) 
Este cenário estudado de acordo com os entrevistados define que a tecnologia BIM tem mais enfoque na modelagem computacional, apresentando números mais expressivos de $60 \%$, de acordo com a figura abaixo:

Figura 3 - Aspecto que o BIM mais enfoca,

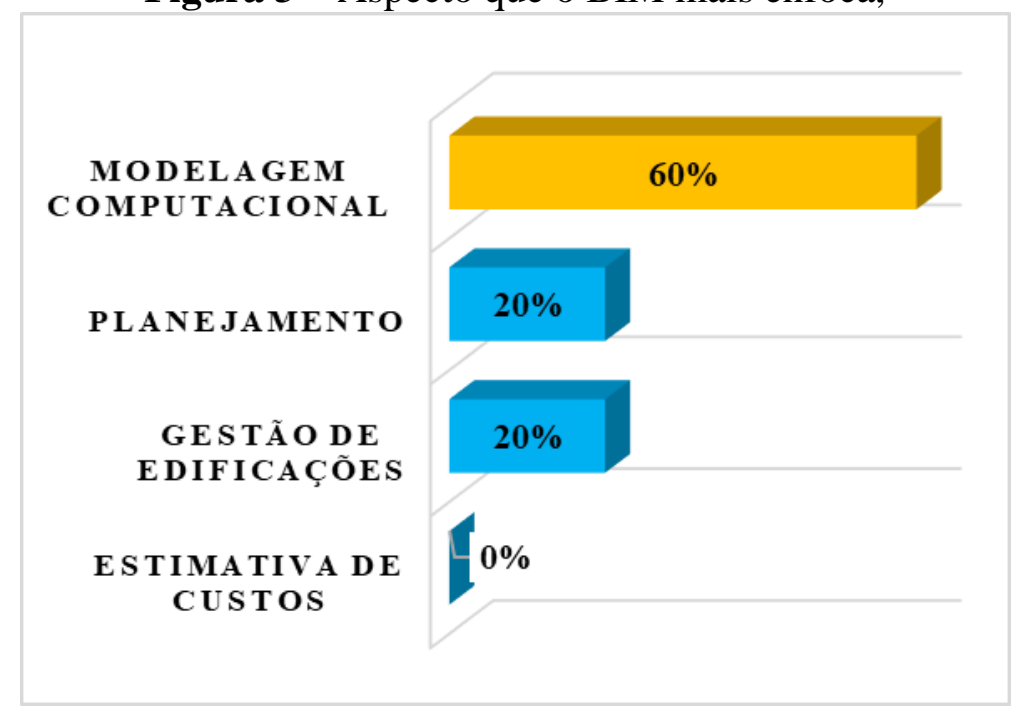

Fonte - Autores (2018)

Organizando o somatório de resultados de todos os entrevistados nos problemas de compatibilização das combinações de projetos propostas em ordem crescente de valor. Notase que $30,77 \%$ de todos os entrevistados afirmaram que já passaram por problemas com a combinação do projeto Estrutural com o projeto de Instalações Sanitárias. Com isso, concluise que o ponto crítico da problemática está localizado nessa combinação. As combinações do projeto Estrutural com de Instalações Hidráulicas, Arquitetônico com Estrutural e Arquitetônico com Instalações Sanitárias também possuem números muito expressivos de problemas, com 23,08\%, 15,38\% e 15,38\% respectivamente. Além disso, a combinação do projeto Arquitetônico com Instalações Hidráulicas, Estrutural com Instalações Elétricas e Arquitetônico com Instalações Elétricas aparecem com números de menor expressão, com $7,69 \%, 7,69 \%$ e $0,00 \%$ respectivamente, ou seja, isso mostra que a compatibilização de projetos não é efetuada de maneira correta na maioria dos casos, referente aos dados acima mencionados, como mostra a figura 4.

No panorama atual analisado, mediante o questionamento aplicado aos colaboradores, a construção civil foi dividida em quatro fases, estrutura, arquitetura, instalações e orçamentação, a etapa na qual a tecnologia BIM deve ter mais aplicação visando minimizar as interferências entre projetos, é a de estrutura, dado que, essa fase obteve números consideráveis de $40 \%$, como a figura 5.

Por meio da organização dos resultados obtidos por intermédio da aplicação de questionário, em ordem crescente de valor, notou-se que quando se trata da associação da tecnologia BIM a otimização de projetos, de acordo com $60 \%$ dos colaboradores entrevistados, é de necessidade extrema enquanto que os outro $40 \%$ entendem que é somente necessário para otimização de projetos, como mostra a figura 6 . 
Figura 4 - Escala de problemas na compatibilização

ESTRUTURAL X INSTALAÇÕES

SA NIT ÁRI A S

ESTRUTURAL X INSTALAÇÕES HIDRÁULICAS

ARQUITETURA X ESTRUTURAL

ARQUITETURA X INSTALAÇÕES SANITÁRIAS

ARQUITETURA X INSTALAÇÕES HI DRÁULICAS

ESTRUTURAL X INSTALAÇÕES ELÉ T RI CAS

ARQUITETURA X INSTALAÇÕES ELÉTRICAS

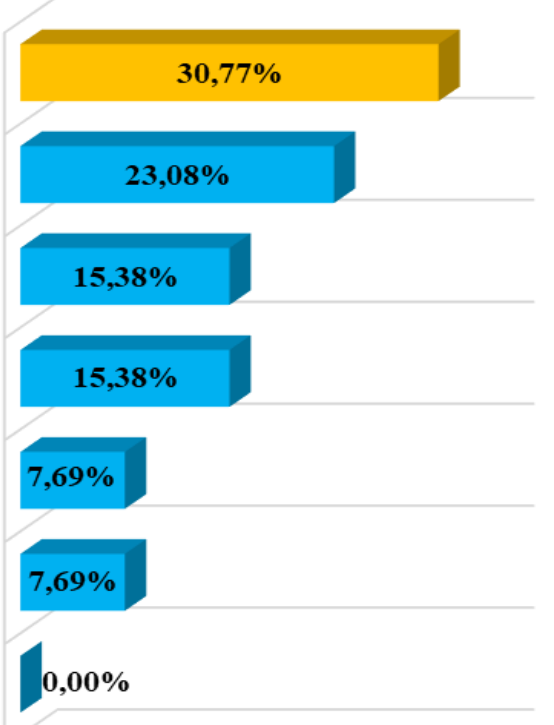

Fonte - Autores (2018)

Figura 5 - Fases da Construção Civil que o Bim deve ser aplicado.

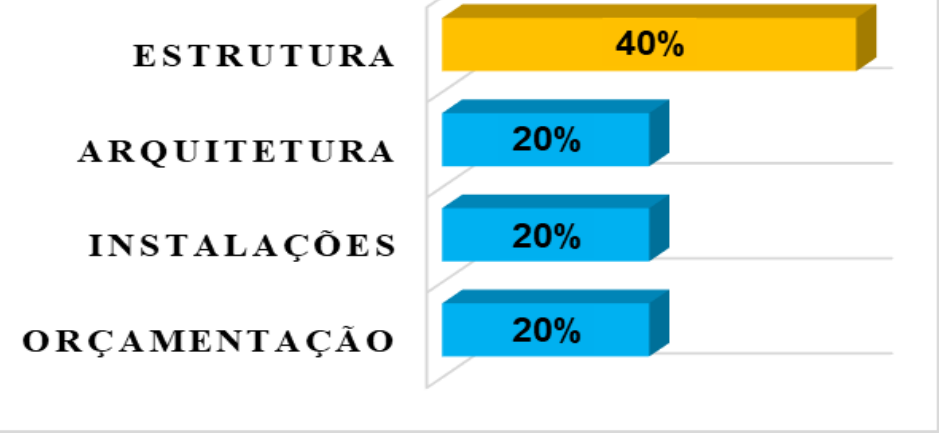

Fonte - Autores (2018)

Figura 6 - Associação do BIM a otimização de projetos.

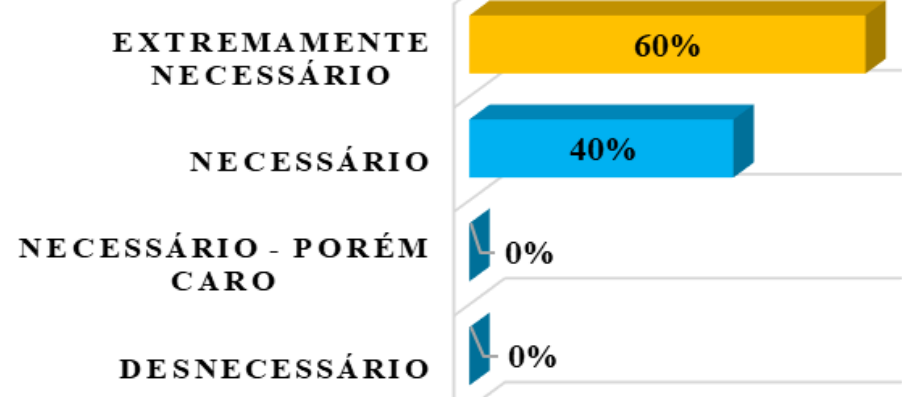

Fonte - Autores (2018) 


\section{CONSIDERAÇÕES FINAIS}

Foi possível analisar o processo de desenvolvimento de projetos e obter informações de como é executada a compatibilização dos mesmos após o apanhado dos resultados oriundos das entrevistas. Tais resultados foram representados em gráficos para auxiliar o entendimento das interferências entre projetos.

De acordo com os resultados obtidos, foi possível constatar que a quantidade de problemas em edificações devido as interferências entre projetos apresentaram números expressivos. Uma forma de reduzir essa problemática, é através da melhor comunicação entre contratado e contratante, visto que, o contratado indique os benefícios que a otimização de projeto realizada antes da fase de execução, reduz consideravelmente o custo final de uma obra. Como Horostecki (2014) explana que "compatibilizar projetos requer investimentos que podem representar de $1 \%$ a $1,5 \%$ do custo da obra, mas gera diminuição de despesas que varia de $5 \%$ a $10 \%$ desse mesmo custo". Além do mais, é aconselhável que mesmo o contratado possuindo vasta experiência no setor construtivo, as normas regulamentadoras devem ser consultadas com frequência.

Ao analisar o tipo de tecnologia utilizada para a compatibilização de projetos, foi constatado que todos entrevistados utilizam um software baseado na tecnologia CAD, mesmo conhecendo outros softwares baseados na tecnologia BIM, foi observado que alguns profissionais ainda não migraram para outro mecanismo por ser conveniente trabalhar em um ambiente que já dominam e julgam ser um sistema mais fácil e acessível para a realização de tal tarefa. Outro fato que pode ser observado no cenário estudado, foi a frequência da aparição de problemas, pois, o software utilizado não possui um processo de compatibilização próprio, sendo a compatibilização realizada no plano bidimensional e de forma mecânica.

Ao avaliar as incompatibilidades entre os principais tipos de projetos abordados neste trabalho, como Arquitetônico, Estrutural, Hidráulico, Sanitário e Elétrico, constatou-se que o ponto crítico está localizado na combinação do projeto Estrutural com o projeto Sanitário. As combinações do projeto Estrutural com o projeto Hidráulico também possuem números muito expressivos. Isso mostra que não é executada a otimização adequada entre projetos de engenharia. Como a estrutura é o conjunto de elementos que sustenta e da estabilidade a edificação, é a etapa na qual as interferências devem ser minimizadas. Vale salientar, mesmo o projeto Arquitetônico não apresentando os índices mais elevados, esse deve ser seguido como principal, pois idealiza como a construção vai ser após seu termino, entretanto, cada projeto deve ser respeitado e executado de acordo com as normas regulamentadoras.

\section{REFERÊNCIAS BIBLIOGRÁFIACAS}

ALVES, C. M. F.; CARVALHO, F. M. S. R.; ROQUE, J. M. G. F.; TEIXEIRA, J. P. M.; PEREIRA, J. P. S.; DEVESA, L. F. S. O que são os BIM. Dissertação de Mestrado Integrado em Engenharia Civil. Faculdade de Engenharia Da Universidade Do Porto. Porto, Portugal.2012.

AYRES, F. C.; SCHEER, S. Diferentes abordagens do uso do CAD no processo de projeto arquitetônico. Workshop Brasileiro de Gestão do Processo de Projetos na Construção de Edifícios. Curitiba. 2007.

BORGES, D. A.; SARMENTO, A. P.; DE CARVALHO, G. B. Projeto arquitetônico para a cidade de catalão/go: diretrizes para a zona bioclimática 6. REEC-Revista Eletrônica de Engenharia Civil, v. 14, n. 1, 2018. 
BORTOLOTTO, M. C. Compatibilização de projetos de uma habitação: Verificação de incompatibilidades no sistema de projetação $2 \mathrm{D}$ e na modelagem $3 \mathrm{D}$. Monografia. UFSC. Florianópolis/SC. 2014.

CAMPESTRINI, T. F. Entendendo BIM - Uma visão do projeto de construção sob o foco da informação. 1. ed. Cuiabá: FABRÍCIO NOGUEIRA COSTA, 2015.

DENCKER, A. F. M. A pesquisa e a interdisciplinaridade no ensino superior: uma experiência no curso de turismo. Tese de Doutorado. 2000.

EASTMAN, C.; TEICHOLZ, P.; SACKS, R.; LISTON, K. Manual de BIM: Um guia de modelagem da informação da construção para arquitetos, engenheiros, gerentes, construtores e incorporadores. Bookman.2014.

FERREIRA, R. C. Os diferentes conceitos adotados entre gerência, coordenação e compatibilização de projeto na construção de edifícios. In: Proceedings of I Workshop Brasileiro Gestão do Processo de Projeto na Construção de Edifícios. 2001.

FLORIO, W. Contribuições do Building Information Modeling no processo de projeto em arquitetura. Encontro de Tecnologia de Informação e Comunicação na Construção Civil. Porto Alegre, 2007.

GEROLlA, G. Carreira: Coordenador de projetos. Téchne-Rev. Pini Web - 190 $^{\mathbf{a}}$ Edição. Nov. 2012. Disponível em: <http://techne17.pini.com.br/engenhariacivil/190/coordenador-de-projetos-com-formacao-generalista-e-dominio-em-288014-1.aspx>. Acesso em: 24 de março. 2018.

GRAZIANO, F. P. Compatibilização de projetos. Instituto de Pesquisas tecnológicas IPT. Dissertação de Mestrado. São Paulo. 2003.

GRILO, L. M. Gestão do processo de projeto no segmento de construção de edifícios por encomenda. Dissertação de Mestrado. Escola Politécnica da Universidade de São Paulo. 391p. 2002.

HELDMAN, K. Project management professional exam - study guide. Indianápolis: Wiley Publishing, 2009.

HOROSTECKI, A. R. N. Compatibilização de projetos de engenharia: Arquitetura em empresas de pequeno porte. UNICSUL. Florianópolis. 2014.

JIANG, X. Developments in cost estimating and scheduling in BIM technology. Tese de Doutorado. Northeastern University. 2011.

LIMEIRA, J.P.S.S.; AYRES, V.C. Compatibilização e coordenação de projetos interdisciplinares em plataforma BIM. Universidade Católica de Brasília. Brasília.2015.

MONTEIRO, A. C. N.; SOBRINHO JR, A. S.; CAVALCANTI, D. S. C.; PEREIRA, E. E. Compatibilização de projetos na construção civil: importância, métodos e ferramentas. Revista Campo do Saber, v. 3, n. 1, 2017.

NASCIMENTO, J. A importância da compatibilização de projetos como fator de redução de custos na construção civil. Revista Especialize On-line IPOG - Goiânia - $7^{\mathbf{a}}$ Edição no 
<http://www.bussinesstour.com.br/uploads/arquivos/64ca01a03c9a31c6fef5ea0686ec6fe8.pdf >. Acesso em: 26 de fev. 2018.

NUNES, G. H.; LEÃO, M. Estudo comparativo de ferramentas de projetos entre o CAD tradicional e a modelagem BIM. Revista de Engenharia, v. 155, n. 55, p. 47-61, 2018.

ORTEGA, L. G.; GEHBAUER, F. Compatibilização de projetos na construção civil. Recife, Projeto competir. 2006.

PICCHI, F. A. Sistemas da qualidade: uso em empresas de construção de edifícios. Tese de Doutorado. Escola Politécnica, Universidade de São Paulo, São Paulo.1993.

RODRÍGUEZ, M. A. A.; HEINECK, L. F. M. Coordenação de projetos: uma experiência de 10 anos dentro de empresas construtoras de médio porte. Simpósio brasileiro de gestão da qualidade e organização do trabalho no ambiente construído, v. 2, 2001.

RODRIGUEZ, M. A. A. Coordenação Técnica de Projetos: Caracterização e Subsídios para sua Aplicação na Gestão do Processo de Projeto de Edificações. Tese de Doutorado. UFSC. Florianópolis/SC. 2005.

RUSCHEL, R. C.; BIZELLO, S. A. Avaliação de sistemas CAD: o processo de projeto em arquitetura da teoria à tecnologia. São Paulo: oficina de Textos. 2011.

SALGADO, M. S. Gestão do Processo de Projeto na Construção do Edifício-revisão 1. Apostila. GEPARQ-Grupo de Pesquisa Gestão em Projetos de Arquitetura, Programa de Pós-Graduação em Arquitetura, Faculdade de Arquitetura e Urbanismo, Universidade Federal do Rio de Janeiro, 2007.

SIMÕES, D. G. Manutenção de edifícios apoiada no modelo BIM. Tese de Mestrado. Instituto Superior Técnico de Lisboa. Portugal. 2013.

SOUSA, H. D. C. Modelação em BIM de armaduras de betão armado de um edifício: Análise da sua contribuição para processos de medição e orçamentação mais eficientes. Tese de Doutorado. Universidade do Minho, escola de engenharia. 2013.

TAVARES JUNIOR, W. Desenvolvimento de um modelo para compatibilização das interfaces entre especialidades do projeto de edificações em empresas construtoras de pequeno porte. 2001. 128 f. 2001. Tese de Doutorado. Dissertação de Mestrado. Programa de Pós-graduação em Engenharia de Produção. UFSC. Florianópolis/SC.2001.

VASCONCELOS, T. M. N. R. F. Building Information Model-Avaliação do seu potencial como solução para os principais atrasos e desperdícios na construção portuguesa. Tese de Doutorado. Faculdade de Ciências e Tecnologia. Universidade nova de Lisboa.Portugal.2010.

VICO Softwares - 5D BIM. Vico Software web Page. Disponível em: <http://www.vicosoftware.com/what-is5D-BIM/tabid/88207/Default.aspx>. Acesso em: $07 \mathrm{de}$ março. 2018. 